\title{
Nutritional Situation of the Elderly in the European Union: Data of the European Nutrition and Health Report (2004)
}

\author{
Elisabeth Fabian Ibrahim Elmadfa \\ Department of Nutritional Sciences, University of Vienna, Vienna, Austria
}

\section{Key Words}

Europe $\cdot$ Elderly $\cdot$ Nutrient intake

\begin{abstract}
Background: The European Nutrition and Health Report contains data about the nutritional situation of elderly of 11 EU countries. Methods: The assessment of the energy and nutrient intake was performed using different methods: 24 hour recall, (semiquantitative) FFQ, 4/7-day-weighed record, personal interview, HBS. Results: Compared to adults of the same country, the energy intake of elderly was on average lower. In most EU countries, the mean proportion of protein intake was within the recommended range while the share of carbohydrates was considerable low. The mean fat intake was remarkably high, the dietary fatty acid pattern predominantly imbalanced and the intake of cholesterol exceeded $300 \mathrm{mg} /$ day in most European elderly. The average intake of vitamin D and folic acid was clearly below 10 and $400 \mu \mathrm{g} /$ day, respectively, in elderly of all countries. Sodium intake was quite high in several participating countries, whereas calcium intake remained below $800 \mathrm{mg} / \mathrm{d}$ in all investigated states. The supply of vitamin $A / C$, thiamine, niacin, cobalamin, and the elements: magnesium, iron, zinc, copper, and manganese could generally be regarded as sufficient. Only in some countries the intake of these nutrients had to be assessed as unsaturated. Conclusion: Data analysis showed that the proportions of macronutrient intake of
\end{abstract}

European elderly are not balanced. Apart from vitamin $D$, calcium, folate, iodine, and sodium the mean intake of micronutrients could be regarded as acceptable.

Copyright $\odot 2008$ S. Karger AG, Basel

\section{Introduction}

The European Nutrition and Health Report is the first comprehensive assessment of the present status of nutrition and health in Europe, and of the available resources and requirements for compatible and representative data from different countries. Thirteen EU member states (Austria, Belgium, Denmark, Finland, France, Germany, Greece, Italy, Portugal, Spain, Sweden, the United Kingdom, and Hungary) as well as Norway have participated in this project, pursuing three main goals: (1) the compilation of available nutrient intake and health data, (2) the identification of major nutrition and health problems in the participating countries and EU regions, and (3) the identification of problems concerning the methods and compatibility of data collection [1]. The European Nutrition and Health Report contains important information concerning the energy and nutrient intake of different population groups (children, adolescents, adults, elderly) in many European countries. National nutrient intake data of the elderly are presented from 11 countries.

\section{KARGER}

Fax +4161306 1234 E-Mail karger@karger.ch www.karger.com

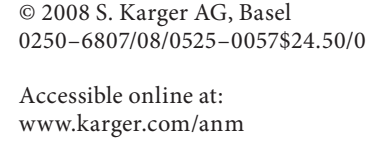


Table 1. Intake of energy and macronutrients (mean \pm SD) in European elderly

\begin{tabular}{|c|c|c|c|c|c|c|c|c|c|c|c|c|}
\hline \multirow[t]{2}{*}{ Country } & \multirow{2}{*}{$\begin{array}{l}\text { Age } \\
\text { years }\end{array}$} & \multirow[t]{2}{*}{$\mathrm{n}$} & \multirow{2}{*}{$\begin{array}{l}\text { Energy } \\
\text { MJ }\end{array}$} & \multirow{2}{*}{$\begin{array}{l}\text { Protein } \\
\% \mathrm{E}\end{array}$} & \multicolumn{2}{|c|}{ Carbohydrate, \%E } & \multirow{2}{*}{$\begin{array}{l}\text { Dietary } \\
\text { fiber } \\
\mathrm{g}\end{array}$} & \multirow{2}{*}{$\begin{array}{l}\text { Fat } \\
\% \mathrm{E}\end{array}$} & \multirow{2}{*}{$\begin{array}{l}\text { SFA } \\
\% \mathrm{E}\end{array}$} & \multirow{2}{*}{$\begin{array}{l}\text { MUFA } \\
\% \mathrm{E}\end{array}$} & \multirow{2}{*}{$\begin{array}{l}\text { PUFA } \\
\% \mathrm{E}\end{array}$} & \multirow{2}{*}{$\begin{array}{l}\text { Choles- } \\
\text { terol } \\
\text { mg }\end{array}$} \\
\hline & & & & & total & sucrose & & & & & & \\
\hline \multicolumn{13}{|l|}{ Males } \\
\hline \multirow[t]{4}{*}{ Austria } & $55-64$ & 21 & $9.0 \pm 3.2$ & $16 \pm 5$ & $45 \pm 9$ & $8 \pm 5$ & $20 \pm 7$ & $36 \pm 6$ & $16 \pm 2$ & $15 \pm 2$ & $5 \pm 2$ & $466 \pm 232$ \\
\hline & $65-74$ & 22 & $8.7 \pm 2.3$ & $16 \pm 5$ & $45 \pm 10$ & $8 \pm 4$ & $23 \pm 9$ & $36 \pm 7$ & $17 \pm 3$ & $14 \pm 2$ & $5 \pm 2$ & $323 \pm 113$ \\
\hline & $75-84$ & 22 & $8.7 \pm 2.3$ & $16 \pm 5$ & $42 \pm 6$ & $7 \pm 3$ & $22 \pm 9$ & $40 \pm 6$ & $20 \pm 3$ & $15 \pm 2$ & $5 \pm 2$ & $377 \pm 119$ \\
\hline & $\geq 85$ & 22 & $7.4 \pm 2.4$ & $14 \pm 5$ & $44 \pm 11$ & $11 \pm 15$ & $15 \pm 5$ & $40 \pm 8$ & $20 \pm 3$ & $15 \pm 2$ & $5 \pm 2$ & $354 \pm 155$ \\
\hline Belgium & $70-75$ & 120 & $10.4 \pm 2.9$ & $13 \pm 3$ & $41 \pm 7$ & n.a. & n.a. & $41 \pm 7$ & $16 \pm 4$ & $15 \pm 5$ & $9 \pm 5$ & $318 \pm 140$ \\
\hline \multirow[t]{2}{*}{ Denmark } & $65-74$ & 122 & 10.9 & 14 & 41 & $7^{4}$ & 25 & 39 & 17 & 12 & 5 & 466 \\
\hline & $75-80$ & 64 & 10.5 & 13 & 41 & $7^{4}$ & 20 & 38 & 16 & 11 & 6 & 415 \\
\hline Germany & $\geq 65$ & 1,509 & 12.3 & 13 & 45 & 12 & 31 & 36 & 14 & 12 & 7 & 358 \\
\hline \multirow[t]{2}{*}{ Greece } & $65-74$ & 2,377 & $8.5 \pm 2.5$ & $14 \pm 2$ & $40 \pm 6$ & n.a. & n.a. & $44 \pm 5$ & $12 \pm 3$ & $21 \pm 4$ & $7 \pm 3$ & n.a. \\
\hline & $\geq 75$ & 340 & $7.9 \pm 2.3$ & $14 \pm 2$ & $41 \pm 6$ & n.a. & n.a. & $44 \pm 6$ & $12 \pm 3$ & $21 \pm 4$ & $6 \pm 3$ & n.a. \\
\hline \multirow[t]{2}{*}{ Hungary } & $\geq 60$ & 55 & $11.8 \pm 3.5$ & $15 \pm 2$ & $44 \pm 6$ & $14 \pm 6$ & n.a. & $39 \pm 6$ & $15 \pm 6$ & $16 \pm 7$ & $4 \pm 2$ & $474 \pm 212$ \\
\hline & $70-75$ & 21 & $11.2 \pm 4.7$ & $12 \pm 2$ & $49 \pm 12$ & n.a. & n.a. & $32 \pm 8$ & $12 \pm 3$ & $12 \pm 3$ & $5 \pm 3$ & $333 \pm 200$ \\
\hline Italy $^{1}$ & $\geq 65$ & n.a. & $8.2 \pm 2.0$ & $16 \pm 4$ & $46 \pm 13$ & $14 \pm 5$ & $20 \pm 7$ & $32 \pm 10$ & $10 \pm 3$ & $13 \pm 5$ & $5 \pm 3$ & $291 \pm 115$ \\
\hline Norway & $60-79$ & n.a. & 8.8 & 16 & 52 & 8 & 25 & 30 & 12 & 11 & 5 & 292 \\
\hline Portugal & $70-75$ & 77 & $8.0 \pm 2.5$ & $16.4 \pm 2.9$ & $52.4 \pm 9.4$ & n.a. & n.a. & $25.7 \pm 5.7$ & $8.4 \pm 2.6$ & $10.5 \pm 2.6$ & $3.8 \pm 1.3$ & $223 \pm 85$ \\
\hline Spain & $65-75$ & 382 & 7.8 & 17 & 46 & n.a. & 19 & 33 & 10 & 15 & 5 & 343 \\
\hline \multirow[t]{3}{*}{ UK } & $65-74$ & 313 & $8.2 \pm 2.0$ & $15 \pm 3^{2}$ & $49 \pm 7^{2}$ & $13 \pm 7^{2}$ & $20 \pm 8^{3}$ & $35 \pm 7^{2}$ & $14 \pm 4^{2}$ & $11 \pm 2^{2}$ & $6 \pm 2^{2}$ & $302 \pm 122$ \\
\hline & $75-84$ & 360 & $7.8 \pm 1.9$ & $15 \pm 3^{2}$ & $51 \pm 7^{2}$ & $15 \pm 8^{2}$ & $18 \pm 7^{3}$ & $35 \pm 6^{2}$ & $15 \pm 3^{2}$ & $11 \pm 2^{2}$ & $5 \pm 2^{2}$ & $288 \pm 130$ \\
\hline & $\geq 85$ & 178 & $7.5 \pm 2.0$ & $14 \pm 3^{2}$ & $51 \pm 7^{2}$ & $17 \pm 8^{2}$ & $16 \pm 6^{3}$ & $36 \pm 5^{2}$ & $16 \pm 3^{2}$ & $11 \pm 2^{2}$ & $5 \pm 2^{2}$ & $283 \pm 119$ \\
\hline \multicolumn{13}{|l|}{ Females } \\
\hline \multirow[t]{4}{*}{ Austria } & $55-64$ & 180 & $7.5 \pm 2.3$ & $16 \pm 4$ & $47 \pm 9$ & $9 \pm 5$ & $21 \pm 8$ & $36 \pm 9$ & $17 \pm 4$ & $14 \pm 3$ & $5 \pm 3$ & $299 \pm 172$ \\
\hline & $65-74$ & 121 & $7.5 \pm 2.0$ & $16 \pm 5$ & $46 \pm 8$ & $9 \pm 5$ & $20 \pm 7$ & $37 \pm 7$ & $17 \pm 3$ & $14 \pm 2$ & $6 \pm 2$ & $325 \pm 222$ \\
\hline & $75-84$ & 160 & $7.4 \pm 1.9$ & $16 \pm 5$ & $45 \pm 9$ & $9 \pm 5$ & $18 \pm 7$ & $38 \pm 8$ & $19 \pm 4$ & $14 \pm 2$ & $5 \pm 2$ & $300 \pm 130$ \\
\hline & $>84$ & 93 & $7.1 \pm 2.0$ & $16 \pm 4$ & $43 \pm 9$ & $9 \pm 5$ & $16 \pm 6$ & $40 \pm 8$ & $20 \pm 3$ & $15 \pm 2$ & $5 \pm 2$ & $337 \pm 225$ \\
\hline Belgium & $70-75$ & 100 & $8.4 \pm 1.9$ & $14 \pm 3$ & $43 \pm 8$ & n.a. & n.a. & $42 \pm 8$ & $17 \pm 5$ & $15 \pm 4$ & $8 \pm 4$ & $273 \pm 107$ \\
\hline \multirow[t]{2}{*}{ Denmark } & $65-74$ & 103 & 8.5 & 16 & 43 & 84 & 19 & 39 & 17 & 11 & 5 & 382 \\
\hline & $75-80$ & 44 & 8.2 & 14 & 45 & 94 & 18 & 38 & 17 & 11 & 5 & 387 \\
\hline Germany & $\geq 65$ & 1,690 & 9.6 & 13 & 46 & 15 & 23 & 38 & 16 & 13 & 6 & 328 \\
\hline Greece & $65-74$ & 3,439 & $6.8 \pm 2.0$ & $14 \pm 2$ & $42 \pm 6$ & n.a. & n.a. & $45 \pm 5$ & $12 \pm 3$ & $22 \pm 4$ & $7 \pm 3$ & n.a. \\
\hline & $\geq 75$ & 344 & $6.4 \pm 2.2$ & $14 \pm 2$ & $42 \pm 6$ & n.a. & n.a. & $45 \pm 5$ & $12 \pm 3$ & $21 \pm 4$ & $7 \pm 3$ & n.a. \\
\hline Hungary & $\geq 55$ & 105 & $9.7 \pm 2.6$ & $15 \pm 2$ & $47 \pm 7$ & $17 \pm 6$ & n.a. & $37 \pm 6$ & $14 \pm 5$ & $15 \pm 6$ & $4 \pm 2$ & $375 \pm 169$ \\
\hline & $70-75$ & 26 & $9.7 \pm 2.9$ & $14 \pm 2$ & $51 \pm 7$ & n.a. & n.a. & $35 \pm 7$ & $13 \pm 3$ & $12 \pm 3$ & $6 \pm 2$ & $328 \pm 108$ \\
\hline Italy $^{1}$ & $\geq 65$ & n.a. & $8.2 \pm 2.0$ & $16 \pm 4$ & $46 \pm 13$ & $14 \pm 5$ & $20 \pm 7$ & $32 \pm 10$ & $10 \pm 3$ & $13 \pm 5$ & $5 \pm 3$ & $291 \pm 115$ \\
\hline Norway & $60-79$ & n.a. & 7.1 & 17 & 53 & 8 & 22 & 29 & 12 & 11 & 5 & 222 \\
\hline Portugal & $70-75$ & 80 & $5.5 \pm 1.6$ & $17 \pm 3$ & $56.2 \pm 7.3$ & n.a. & n.a. & $27.7 \pm 6.2$ & $9.2 \pm 2.8$ & $11.1 \pm 3.1$ & $4.4 \pm 1.5$ & $178 \pm 87$ \\
\hline Spain & $65-75$ & 429 & 6.5 & 18 & 47 & n.a. & 16 & 34 & 11 & 15 & 4 & 304 \\
\hline UK & $65-74$ & 278 & $6.1 \pm 1.5$ & $16 \pm 4^{2}$ & $50 \pm 7^{2}$ & $12 \pm 6^{2}$ & $17 \pm 6^{3}$ & $36 \pm 6^{2}$ & $15 \pm 4^{2}$ & $11 \pm 2^{2}$ & $6 \pm 2^{2}$ & $224 \pm 95$ \\
\hline & $75-84$ & 303 & $6.1 \pm 1.6$ & $16 \pm 3^{2}$ & $51 \pm 6^{2}$ & $14 \pm 7^{2}$ & $15 \pm 5^{3}$ & $36 \pm 6^{2}$ & $15 \pm 4^{2}$ & $11 \pm 2^{2}$ & $6 \pm 2^{2}$ & $230 \pm 96$ \\
\hline & $\geq 85$ & 301 & $6.1 \pm 1.6$ & $14 \pm 3^{2}$ & $52 \pm 7^{2}$ & $16 \pm 8^{2}$ & $14 \pm 5^{3}$ & $37 \pm 6^{2}$ & $16 \pm 4^{2}$ & $11 \pm 2^{2}$ & $5 \pm 2^{2}$ & $228 \pm 102$ \\
\hline Reference & & & & $10-15^{* *}$ & $>55$ & $<10^{* *}$ & $>25$ & $<30$ & $<10$ & & $6-10$ & $<300^{* *}$ \\
\hline
\end{tabular}

${ }^{1}$ Men and women. ${ }^{2} \%$ of total food energy (excluding alcohol). ${ }^{3}$ Nonstarchy polysaccharides. ${ }^{4}$ Added sucrose.

* Eurodiet, 2000; ** WHO, 2003. n.a. = Not available.

\section{Methods}

The assessment of the nutritional intake of European elderly was performed using different methods: 24-hour recalls, (semiquantitative) Food Frequency Questionnaires (FFQ), 4/7-dayweighed records, personal interviews, and Household Budget Surveys (HBS).

\section{Results}

\section{Energy and Macronutrients}

In comparison to younger adults of the same country, the energy intake of elderly was on average lower. Except for Spanish and Norwegian women, the mean proportion of protein in total energy intake was within the recommended range in most countries, or slightly above (ta- 
Table 2. Intake (mean \pm SD) of selected vitamins in European elderly

\begin{tabular}{|c|c|c|c|c|c|c|c|c|c|c|c|c|}
\hline Country & $\begin{array}{l}\text { Age } \\
\text { years }\end{array}$ & $\mathrm{n}$ & $\begin{array}{l}\text { Vitamin } \\
\mathrm{A}^{1} \\
\mathrm{mg}\end{array}$ & $\begin{array}{l}\text { Vitamin } \\
\mathrm{D} \\
\mu \mathrm{g}\end{array}$ & $\begin{array}{l}\text { Vitamin } \\
\mathrm{E}^{2} \\
\mathrm{mg}\end{array}$ & $\begin{array}{l}\text { Thia- } \\
\text { mine } \\
\text { mg }\end{array}$ & $\begin{array}{l}\text { Ribo- } \\
\text { flavin } \\
\mathrm{mg}\end{array}$ & $\begin{array}{l}\mathrm{Niacin}^{3} \\
\mathrm{mg}\end{array}$ & $\begin{array}{l}\text { Vitamin } \\
\mathrm{B}_{6} \\
\mathrm{mg}\end{array}$ & $\begin{array}{l}\text { Folate }^{4} \\
\mu \mathrm{g}\end{array}$ & $\begin{array}{l}\text { Cobal- } \\
\text { amin } \\
\mu \mathrm{g}\end{array}$ & $\begin{array}{l}\text { Ascorbic } \\
\text { acid } \\
\text { mg }\end{array}$ \\
\hline \multicolumn{13}{|l|}{ Males } \\
\hline \multirow[t]{4}{*}{ Austria } & $55-64$ & 21 & $1.5 \pm 1.6$ & $5.0 \pm 5.7$ & $9.9 \pm 4.2$ & $1.4 \pm 0.7$ & $1.5 \pm 0.8$ & $31 \pm 13$ & $1.6 \pm 0.6$ & $234 \pm 92$ & $5.3 \pm 3.4$ & $97 \pm 85$ \\
\hline & $65-74$ & 22 & $1.3 \pm 1.1$ & $5.1 \pm 5.3$ & $9.0 \pm 3.1$ & $1.2 \pm 0.4$ & $1.5 \pm 0.4$ & $27 \pm 7$ & $1.4 \pm 0.4$ & $232 \pm 70$ & $4.9 \pm 2.6$ & $113 \pm 104$ \\
\hline & $75-84$ & 22 & $1.2 \pm 0.8$ & $3.4 \pm 2.2$ & $9.3 \pm 4.1$ & $1.2 \pm 0.4$ & $1.4 \pm 0.3$ & $28 \pm 8$ & $1.5 \pm 0.5$ & $232 \pm 80$ & $5.3 \pm 3.2$ & $109 \pm 86$ \\
\hline & $\geq 85$ & 22 & $0.9 \pm 0.4$ & $3.4 \pm 3.1$ & $6.2 \pm 2.9$ & $1.0 \pm 0.4$ & $1.3 \pm 0.5$ & $23 \pm 9$ & $1.1 \pm 0.4$ & $174 \pm 61$ & $4.0 \pm 1.8$ & $93 \pm 133$ \\
\hline \multirow[t]{2}{*}{ Denmark } & $65-74$ & 122 & 1.6 & 4.1 & 9.2 & 1.3 & 1.9 & 36 & 1.7 & 301 & 6.8 & 79 \\
\hline & $75-80$ & 64 & 1.4 & 3.7 & 10.8 & 1.2 & 1.7 & 29 & 1.5 & 259 & 5.7 & 69 \\
\hline Germany & $\geq 65$ & 1,509 & 1.7 & 4.9 & 19.2 & 1.7 & 1.9 & 39 & 2.4 & 286 & 7.7 & 144 \\
\hline \multirow[t]{2}{*}{ Hungary } & $\geq 60$ & 55 & $0.8 \pm 0.6$ & n.a. & $7.2 \pm 3.4$ & $1.1 \pm 0.4$ & $1.4 \pm 0.5$ & $19 \pm 8$ & $2.0 \pm 0.7$ & n.a. & n.a. & $91 \pm 64$ \\
\hline & $70-75$ & 21 & $0.3 \pm 0.1$ & n.a. & n.a. & $0.6 \pm 0.5$ & $0.9 \pm 0.4$ & n.a. & $1.3 \pm 0.8$ & n.a. & n.a. & $66 \pm 31$ \\
\hline Italy $^{5}$ & $\geq 65$ & n.a. & $1.2 \pm 1.2$ & $2.5 \pm 1.8$ & $10.9 \pm 4.4$ & $1.0 \pm 0.3$ & $1.5 \pm 0.5$ & $18 \pm 5$ & $1.9 \pm 0.5$ & $295 \pm 166$ & n.a. & $109 \pm 60$ \\
\hline Norway & $60-79$ & n.a. & 1.6 & 5.8 & n.a. & 1.5 & 1.7 & 17 & n.a. & n.a. & n.a. & 117 \\
\hline Portugal $^{6}$ & $70-75$ & 77 & 0.64 & n.a. & n.a. & 1.08 & 1.65 & n.a. & 1.38 & n.a. & n.a. & 87 \\
\hline Spain & $65-75$ & 382 & 1.2 & 4.1 & 7.9 & 1.1 & 1.5 & 19 & 1.8 & 311 & 7.4 & 124 \\
\hline \multirow[t]{3}{*}{ UK } & $65-74$ & 313 & $0.9 \pm 1.6$ & $4.6 \pm 3.7$ & $10.2 \pm 9.8$ & $1.6 \pm 0.6$ & $1.8 \pm 0.8$ & $33 \pm 9$ & $2.2 \pm 0.8$ & $283 \pm 108$ & $6.3 \pm 5.7$ & $74 \pm 62$ \\
\hline & $75-84$ & 360 & $0.8 \pm 1.6$ & $4.1 \pm 2.9$ & $8.7 \pm 5.1$ & $1.4 \pm 0.6$ & $1.7 \pm 0.8$ & $30 \pm 9$ & $2.0 \pm 0.7$ & $248 \pm 103$ & $5.3 \pm 5.8$ & $61 \pm 51$ \\
\hline & $\geq 85$ & 178 & $0.8 \pm 1.1$ & $3.7 \pm 2.4$ & $7.9 \pm 9.1$ & $1.4 \pm 0.5$ & $1.8 \pm 0.8$ & $28 \pm 9$ & $1.8 \pm 0.7$ & $238 \pm 96$ & $5.0 \pm 4.3$ & $59 \pm 80$ \\
\hline \multicolumn{2}{|c|}{ Reference values* } & & 0.7 & $10^{* *}$ & - & 1.1 & 1.6 & 18 & 1.5 & $200 / 400^{* *}$ & 1.4 & 45 \\
\hline \multicolumn{13}{|l|}{ Females } \\
\hline \multirow[t]{4}{*}{ Austria } & $55-64$ & 180 & $1.1 \pm 0.9$ & $3.4 \pm 4.4$ & $8.6 \pm 5.2$ & $1.2 \pm 0.5$ & $1.4 \pm 0.6$ & $25 \pm 8$ & $1.4 \pm 0.6$ & $223 \pm 88$ & $3.9 \pm 2.1$ & $109 \pm 73$ \\
\hline & $65-74$ & 121 & $1.2 \pm 1.2$ & $4.7 \pm 6.2$ & $9.3 \pm 5.5$ & $1.1 \pm 0.5$ & $1.5 \pm 0.6$ & $25 \pm 10$ & $1.4 \pm 0.6$ & $210 \pm 68$ & $4.4 \pm 2.9$ & $98 \pm 66$ \\
\hline & $75-84$ & 160 & $1.1 \pm 1.1$ & $3.6 \pm 6.1$ & $7.2 \pm 3.2$ & $1.0 \pm 0.4$ & $1.4 \pm 0.5$ & $24 \pm 8$ & $1.3 \pm 0.5$ & $189 \pm 67$ & $4.1 \pm 2.5$ & $87 \pm 63$ \\
\hline & $>84$ & 93 & $1.0 \pm 1.0$ & $3.1 \pm 3.5$ & $6.3 \pm 2.7$ & $0.9 \pm 0.4$ & $1.3 \pm 0.6$ & $23 \pm 8$ & $1.2 \pm 0.5$ & $166 \pm 60$ & $3.9 \pm 2.3$ & $71 \pm 62$ \\
\hline \multirow[t]{2}{*}{ Denmark } & $65-74$ & 103 & 1.6 & 3.3 & 7.8 & 1.1 & 1.6 & 29 & 1.4 & 261 & 6.1 & 79 \\
\hline & $75-80$ & 44 & 1.6 & 3.2 & 7.8 & 1.0 & 1.6 & 24 & 1.3 & 273 & 6.2 & 77 \\
\hline Germany & $\geq 65$ & 1,690 & 1.5 & 3.1 & 13.1 & 1.2 & 1.6 & 30 & 1.6 & 231 & 5.5 & 123 \\
\hline \multirow[t]{2}{*}{ Hungary } & $\geq 55$ & 105 & $0.8 \pm 0.6$ & n.a. & $7.1 \pm 3.7$ & $1.0 \pm 0.4$ & $1.2 \pm 0.4$ & $17 \pm 6$ & $1.8 \pm 0.6$ & n.a. & $3.8 \pm 4.8$ & $94 \pm 62$ \\
\hline & $70-75$ & 26 & $0.4 \pm 0.2$ & n.a. & n.a. & $0.8 \pm 0.5$ & $1.2 \pm 0.7$ & n.a. & n.a. & n.a. & n.a. & $56 \pm 34$ \\
\hline Italy $^{5}$ & $\geq 65$ & n.a. & $1.2 \pm 1.2$ & $2.5 \pm 1.8$ & $10.9 \pm 4.4$ & $1.0 \pm 0.3$ & $1.5 \pm 0.5$ & $18 \pm 5$ & n.a. & n.a. & n.a. & $109 \pm 60$ \\
\hline Norway & $60-79$ & n.a. & 1.6 & 4.0 & n.a. & 1.2 & 1.5 & 14 & n.a. & n.a. & n.a. & 123 \\
\hline Portugal $^{6}$ & $70-75$ & 80 & 0.51 & n.a. & n.a. & 0.62 & 1.24 & n.a. & 0.93 & n.a. & n.a. & 62 \\
\hline Spain & $65-75$ & 429 & 1.1 & 2.9 & n.a. & 1.0 & 1.6 & 17 & 1.5 & 272 & 6.2 & 118 \\
\hline \multirow[t]{3}{*}{ UK } & $65-74$ & 278 & $0.8 \pm 1.2$ & $3.4 \pm 2.7$ & $11.1 \pm 37.6$ & $1.7 \pm 6.0$ & $1.9 \pm 6.1$ & $27 \pm 10$ & $2.3 \pm 6.9$ & $226 \pm 92$ & $4.6 \pm 4.2$ & $73 \pm 58$ \\
\hline & $75-84$ & 303 & $0.8 \pm 1.2$ & $3.4 \pm 2.7$ & $9.1 \pm 35.6$ & $1.3 \pm 0.7$ & $1.6 \pm 0.9$ & $25 \pm 10$ & $1.7 \pm 0.9$ & $213 \pm 114$ & $3.9 \pm 3.3$ & $58 \pm 53$ \\
\hline & $\geq 85$ & 301 & $0.7 \pm 1.4$ & $3.0 \pm 2.1$ & $7.1 \pm 10.6$ & $1.1 \pm 0.4$ & $1.4 \pm 0.6$ & $23 \pm 8$ & $1.5 \pm 0.7$ & $189 \pm 86$ & $4.3 \pm 3.9$ & $52 \pm 63$ \\
\hline \multicolumn{2}{|c|}{ Reference values* } & & 0.6 & $10^{* *}$ & - & 0.9 & 1.3 & 14 & 1.1 & $200 / 400^{* *}$ & 1.4 & 45 \\
\hline
\end{tabular}

\footnotetext{
${ }^{1}$ Retinol equivalent $\left(=1 \mathrm{mg}\right.$ all-trans retinol $=6 \mathrm{mg}$ all-trans $\beta$-carotene $=12 \mathrm{mg}$ other carotenoids). ${ }^{2} \mathrm{RRR}$ - $\alpha$-Tocopherol equivalent $(=\mathrm{mg} \alpha$-tocopherol $+\mathrm{mg} \beta$-tocopherol $\times 0.5+\mathrm{mg} \gamma$-tocopherol $\times 0.25+\mathrm{mg} \alpha$-tocotrienol $\times 0.33) .{ }^{3}$ Niacin equivalent $(=1 \mathrm{mg}$ niacin $=60 \mathrm{mg}$ tryptophan $) .{ }^{4}$ Folate equivalent $\left(=1 \mu \mathrm{g}\right.$ food folate $=0.5 \mu \mathrm{g}$ folic acid (PGA) $=0.6 \mu \mathrm{g}$ folic acid taken with meals). ${ }^{5}$ Preformed retinol. ${ }^{6}$ Values corresponded to the median (P50).

* SCF, 1993; ** Eurodiet, 2000. n.a. = Not available.
}

ble 1). The mean share of carbohydrate was considerably low - the recommended level (RL) of at least $55 \% \mathrm{E}$ [2] was only reached by Portuguese women. The lowest carbohydrate intake was observed in men of Belgium, Denmark, and Greece, the highest in Norwegian, Portuguese and UK elderly and Hungarian women. The carbohydrate intake was on average higher in elderly women than in men. Apart from Germany, the UK, and Hungary the mean intake of sucrose was found to be generally below the rec- ommended level of $10 \% \mathrm{E}$ [3]. The highest consumption of sucrose $(17 \pm 6 \% \mathrm{E})$ could be observed in Hungarian elderly women. The consumption of dietary fiber varied strongly among the participating countries. The lowest intake was found in Austrian and UK (both sexes $\geq 85$ years: $14 \pm 5 \mathrm{~g} /$ day) as well as Spanish (women, 65-75 years) elderly, the highest in Norwegian, Danish, and German men (25-31 g/day). 
Table 3. Dietary intake (mean \pm SD) of selected minerals and trace elements in European elderly

\begin{tabular}{|c|c|c|c|c|c|c|c|c|c|c|}
\hline Country & $\begin{array}{l}\text { Age } \\
\text { years }\end{array}$ & $\mathrm{n}$ & $\begin{array}{l}\text { Sodium } \\
\mathrm{g}\end{array}$ & $\begin{array}{l}\text { Potassium } \\
\mathrm{g}\end{array}$ & $\begin{array}{l}\text { Calcium } \\
\mathrm{mg}\end{array}$ & $\begin{array}{l}\text { Phosphorus } \\
\text { mg }\end{array}$ & $\begin{array}{l}\text { Magnesium } \\
\text { mg }\end{array}$ & $\begin{array}{l}\text { Iron } \\
\text { mg }\end{array}$ & $\begin{array}{l}\text { Zinc } \\
\text { mg }\end{array}$ & $\begin{array}{l}\text { Iodine } \\
\mu g\end{array}$ \\
\hline \multicolumn{11}{|l|}{ Males } \\
\hline \multirow[t]{4}{*}{ Austria } & $55-64$ & 21 & n.a. & $2.6 \pm 1.2$ & $635 \pm 402$ & n.a. & $311 \pm 152$ & $14.2 \pm 5.4$ & $12.7 \pm 7.1$ & $123 \pm 52$ \\
\hline & $65-74$ & 22 & n.a. & $2.8 \pm 1.0$ & $959 \pm 400$ & n.a. & $341 \pm 98$ & $14.7 \pm 4.3$ & $12.5 \pm 3.9$ & $136 \pm 54$ \\
\hline & $75-84$ & 22 & n.a. & $2.5 \pm 0.8$ & $695 \pm 266$ & n.a. & $281 \pm 85$ & $14.4 \pm 6.0$ & $11.8 \pm 3.3$ & $119 \pm 23$ \\
\hline & $\geq 85$ & 22 & n.a. & $1.9 \pm 1.9$ & $642 \pm 642$ & n.a. & $233 \pm 84$ & $10.0 \pm 2.9$ & $9.1 \pm 3.3$ & $102 \pm 34$ \\
\hline \multirow{2}{*}{ Denmark } & $65-74$ & 122 & 3.6 & 3.8 & 954 & 1,641 & 380 & 11.9 & 13.5 & 143 \\
\hline & $75-80$ & 64 & 3.4 & 3.3 & 822 & 1,404 & 334 & 9.7 & 11.1 & 101 \\
\hline Germany & $\geq 65$ & 1,509 & 4.3 & 4.2 & 1,031 & 1,657 & 474 & 17.8 & 13.5 & 127 \\
\hline \multirow[t]{2}{*}{ Hungary } & $\geq 60$ & 55 & $7.4 \pm 1.9$ & $2.9 \pm 0.8$ & $699 \pm 365$ & n.a. & $381 \pm 112$ & $12.4 \pm 3.8$ & n.a. & n.a. \\
\hline & $70-75$ & 21 & n.a. & n.a. & $638 \pm 220$ & n.a. & n.a. & $9.7 \pm 2.8$ & n.a. & n.a. \\
\hline Italy $^{1}$ & $\geq 65$ & n.a. & $5.4 \pm 2.9$ & $3.0 \pm 0.8$ & $845 \pm 263$ & $1,242 \pm 295$ & $199 \pm 56$ & $12.6 \pm 4.0$ & $10.7 \pm 3.1$ & n.a. \\
\hline Norway & $60-79$ & n.a. & n.a. & n.a. & 900 & n.a. & n.a. & 11.0 & n.a. & n.a. \\
\hline Portugal $^{2}$ & $70-75$ & 77 & n.a. & n.a. & 766 & n.a. & n.a. & 12.8 & n.a. & n.a. \\
\hline Spain & $65-75$ & 382 & 1.7 & 3.0 & 714 & 1,218 & 295 & 12.5 & n.a. & n.a. \\
\hline \multirow[t]{3}{*}{ UK } & $65-74$ & 313 & $2.8 \pm 0.9$ & $2.8 \pm 0.8$ & $856 \pm 290$ & $1,261 \pm 340$ & $258 \pm 79$ & $11.5 \pm 6.6$ & $9.0 \pm 2.7$ & $193 \pm 82$ \\
\hline & $75-84$ & 360 & $2.7 \pm 0.9$ & $2.5 \pm 0.7$ & $840 \pm 302$ & $1,179 \pm 331$ & $233 \pm 77$ & $10.6 \pm 3.9$ & $8.4 \pm 2.8$ & $185 \pm 80$ \\
\hline & $\geq 85$ & 178 & $2.6 \pm 0.9$ & $2.3 \pm 0.6$ & $847 \pm 285$ & $1,134 \pm 308$ & $214 \pm 69$ & $10.1 \pm 4.1$ & $8.0 \pm 2.6$ & $177 \pm 73$ \\
\hline \multicolumn{2}{|c|}{ Reference values* } & & $0.6-3.5$ & 3.1 & $700 />800^{* *}$ & 550 & $150-500$ & 9 & 9.5 & 130 \\
\hline \multicolumn{11}{|l|}{ Females } \\
\hline \multirow[t]{4}{*}{ Austria } & $55-64$ & 180 & n.a. & $2.6 \pm 0.9$ & $831 \pm 409$ & n.a. & $286 \pm 98$ & $12.6 \pm 6.6$ & $10.4 \pm 3.3$ & $130 \pm 56$ \\
\hline & $65-74$ & 121 & n.a. & $2.6 \pm 0.9$ & $799 \pm 402$ & n.a. & $288 \pm 101$ & $12.3 \pm 5.0$ & $10.3 \pm 3.7$ & $135 \pm 55$ \\
\hline & $75-84$ & 160 & n.a. & $2.3 \pm 0.8$ & $780 \pm 361$ & n.a. & $271 \pm 110$ & $11.0 \pm 4.1$ & $10.1 \pm 3.1$ & $122 \pm 56$ \\
\hline & $>84$ & 93 & n.a. & $2.1 \pm 0.8$ & $649 \pm 351$ & n.a. & $235 \pm 86$ & $11.1 \pm 7.3$ & $9.2 \pm 3.4$ & $111 \pm 42$ \\
\hline \multirow[t]{2}{*}{ Denmark } & $65-74$ & 103 & 2.8 & 3.2 & 912 & 1,366 & 307 & 9.0 & 11.2 & 111 \\
\hline & $75-80$ & 44 & 2.5 & 2.8 & 864 & 1,264 & 276 & 8.5 & 9.8 & 115 \\
\hline Germany & $\geq 65$ & 1,690 & 3.0 & 3.5 & 886 & 1,276 & 377 & 13.4 & 10.1 & 91 \\
\hline \multirow[t]{2}{*}{ Hungary } & $\geq 55$ & 105 & $6.2 \pm 1.8$ & $2.7 \pm 0.8$ & $613 \pm 264$ & n.a. & $328 \pm 96$ & $10.8 \pm 3.3$ & n.a. & n.a. \\
\hline & $70-75$ & 26 & n.a. & n.a. & $838 \pm 527$ & n.a. & n.a. & $9.8 \pm 5.1$ & n.a. & n.a. \\
\hline Italy $^{1}$ & $\geq 65$ & n.a. & $5.4 \pm 2.9$ & $3.0 \pm 0.8$ & $845 \pm 263$ & $1,242 \pm 295$ & $199 \pm 56$ & $12.6 \pm 4.0$ & $10.7 \pm 3.1$ & n.a. \\
\hline Norway & $60-79$ & n.a. & n.a. & n.a. & 800 & n.a. & n.a. & 9.0 & n.a. & n.a. \\
\hline Portugal $^{2}$ & $70-75$ & 80 & n.a. & n.a. & 548 & n.a. & n.a. & 7.0 & n.a. & n.a. \\
\hline Spain & $65-75$ & 429 & 1.5 & 2.9 & 759 & 1,133 & 250 & 10.1 & n.a. & n.a. \\
\hline \multirow[t]{3}{*}{ UK } & $65-74$ & 278 & $2.1 \pm 0.7$ & $2.3 \pm 0.6$ & $720 \pm 251$ & $1,032 \pm 275$ & $208 \pm 61$ & $9.4 \pm 3.5$ & $7.2 \pm 2.2$ & $154 \pm 62$ \\
\hline & $75-84$ & 303 & $2.1 \pm 0.6$ & $2.1 \pm 0.5$ & $729 \pm 270$ & $983 \pm 268$ & $188 \pm 60$ & $8.4 \pm 3.0$ & $7.0 \pm 2.8$ & $151 \pm 63$ \\
\hline & $\geq 85$ & 301 & $2.0 \pm 0.7$ & $2.0 \pm 0.6$ & $718 \pm 273$ & $940 \pm 278$ & $178 \pm 55$ & $7.9 \pm 2.9$ & $6.5 \pm 2.3$ & $149 \pm 67$ \\
\hline \multicolumn{2}{|c|}{ Reference values* } & & $0.6-3.5$ & 3.1 & $700 />800^{* *}$ & 550 & $150-500$ & 9 & 7 & 130 \\
\hline
\end{tabular}

${ }^{1}$ Men and women. ${ }^{2}$ Values corresponded to the median (P50).

* SCF, 1993; ** Eurodiet, 2000. n.a. = Not available.

The mean fat intake was remarkably high (maximum: Greece $45 \pm 5 \% \mathrm{E}$; minimum: Norway $29-30 \% \mathrm{E}$ ) in European elderly. With exception of Greece, countries with a high fat intake also had a high intake of saturated fatty acids (SFA) (up to 20\%E) [2]. The mean intake of polyunsaturated fatty acids (PUFA) remained below the recommended level of $6 \% \mathrm{E}$, while the intake of cholesterol exceeded the upper level (300 mg/day) [3] in nearly all the participating countries (table 1 ).

\section{Vitamins}

The average intake of vitamin D and folate was clearly below the RLs (10 and $400 \mu \mathrm{g} /$ day) [2] in elderly of all participating countries. Apart from Hungary, the vitamin A supply could be regarded as sufficient [4] in all countries which took part in this study.

With exception of Hungarian (70-75 years), Italian ( $\geq 65$ years), and Austrian (men, $\geq 85$ years) elderly the thiamine intake could on average be assessed as sufficient in all participating counties. Apart from Hungary, the mean riboflavin intake was above the RL in elderly wom- 
en but relatively low in European men. The mean niacin and vitamin $\mathrm{B}_{6}$ intake could be regarded as sufficient in all participating elderly except for Norwegian men and Austrian and Hungarian men, respectively. The supply of cobalamin and ascorbic acid was found to be sufficient in all European elderly (table 2).

\section{Minerals}

While sodium intake was quite high in all participants (maximum: Hungary $7.4 \mathrm{~g} /$ day), the supply of potassium was not sufficient in most European elderly. The calcium intake was below the RL of $800 \mathrm{mg} /$ day [2] in several countries (Austria, Hungary, Spain). Except for Danish and UK women, the supply of iron [4] was good in all participating elderly. The mean zinc intake was found to be low in Austrian ( $\geq 85$ years) and UK elderly. Apart from the UK, iodine intake was on average below the RL of $150 \mu \mathrm{g} /$ day [2] (table 3). The supply of magnesium, copper, and manganese could on average be regarded as sufficient.

\section{Discussion}

The European Health and Nutrition Report 2004 is a compilation of existing, available nutrient intake and health data and provides important information about the nutritional situation of elderly in different EU countries. However, data have to be interpreted with caution because of differences in the assessment methods and partly inadequate size and inhomogeneous age groups used in the participating countries.

Available data of the nutritional situation of elderly Europeans clearly indicate an imbalance in the intake of the macronutrients carbohydrate, fat, and dietary fiber in this population group. Additionally, the quality of the consumed fat has to be assessed as inadequate in most participating countries. Except for Belgium and Greece, the intake of SFAs exceeded the reference value of $10 \% \mathrm{E}$ while that of PUFAs remained below the recommended level of $6 \% \mathrm{E}$. Consequently, the cholesterol intake was generally too high, with the exceptions being Italy, Portugal, Norway, the UK, and female Belgians.

The data evaluated for the minerals phosphorus, iodine, zinc, manganese, copper, and the vitamin cobalamin have been identified to have an alleviated explanatory power because of the incomplete intake data of these nutrients.

In order to obtain well-comparable data for future European Health and Nutrition Reports, the authors of the report strongly advise to improve the data collection by the use of standardized methods for the assessment of nutritional status, including food and nutrient intake. Further, uniformed age, adequately sized groups and educational levels would also help to get comparable data of different EU countries.

Finally, the report points out the necessity of updating reference values for nutrient intake valid for the whole of Europe, also including aspects of health promotion and disease prevention.

\section{Conclusion}

Data assessment showed that the proportions of macronutrient intake of European elderly are not balanced and partly inadequate (low: carbohydrates/dietary fiber; high: fat/SFA/cholesterol). Apart from vitamin D, calcium, folate, sodium, and iodine the mean intake of micronutrients could be regarded as rather acceptable.

\section{Disclosure Statement}

The European Nutrition and Health Report project has been financially supported by the European Commission (EC), Health and Consumer Protection Directorate-General, Directorate C - Public Health and Risk Assessment - within the Health Monitoring Programme (Grant Agreement No. SPC.2002356).

References

Ann Nutr Metab 2008;52(suppl 1):57-61
Elmadfa I, Weichselbaum E, Koenig J, Remaut De Winter AM, Trolle E, Haapala, I, Uusitalo U, Mennen L, Hercberg S, Wolfram G, Trichopoulou A, Naska A, Vassiliki B, Dritsellis E, Rodler I, Zajkás G, Branca F, D’Acapito P, Klepp K-I, Ali-Madar A, De Almeida MDV, Alves E, Rodrigues S, SerraMajem L, Roman B, Sjöström M, Poortvliet E, Margetts B: The European Nutrition and Health Report 2004. Forum Nutrition 58. Basel, Karger, 2005.

2 Eurodiet: Nutrition and Diet for the Healthy plications. Heraklion, University of Crete, School of Medicine, 2000

3 World Health Organization (WHO): Diet, Nutrition and the Prevention of Chronic Diseases. Report of a Joint WHO/FAO Expert Consultation. Geneva, World Health Organization, 2003. of the Scientific Committee for Food. Nutrition and Energy Intakes for the European Community. Commission of the European Communities, Directorate General, 1993. Lifestyles in Europe. Science and Policy Im-

4 Scientific Committee for Food (SCF): Report 